\title{
Large Radius Tracking at the ATLAS Experiment
}

\author{
Margaret Lutz ${ }^{* \dagger}$ \\ University of Massachusetts (US) \\ E-mail: margaret.susan.lutz@cern.ch
}

\begin{abstract}
The standard track reconstruction in the ATLAS detector at the LHC is optimized for tracks which point back towards the primary interaction point and thus have small impact parameters. Many different models beyond the Standard Model include particles with long lifetimes which may decay far from the interaction point, leaving tracks which may have large impact parameters. To reconstruct these tracks with large impact parameters, or large radius tracks, an extra tracking pass is enabled. These proceedings discuss the parameters and performance of this large radius tracking pass, as they are described in ATL-PHYS-PUB-2017-014, and were presented in a poster at EPS-HEP in Venice, Italy.
\end{abstract}

EPS-HEP 2017, European Physical Society conference on High Energy Physics 5-12 July 2017

Venice, Italy

* Speaker.

${ }^{\dagger}$ On behalf of the ATLAS Collaboration. 


\section{Motivation}

Many models of physics beyond the Standard Model include long lived particles. The resulting signatures in these models may include tracks which are highly displaced from the proton-proton interaction point (IP) and have large impact parameters. The standard tracking passes in the ATLAS experiment at the LHC are optimized for tracks which point back towards the IP, and are thus unlikely to reconstruct such displaced tracks. A special tracking pass has been developed and validated in order to increase the likelihood of reconstructing tracks with large impact parameters. This tracking pass and its validation are described in detail in the ATLAS public note ATL-PHYSPUB-2017-014 [1] and were presented in a poster at EPS-HEP 2017.

\section{ATLAS inner detector and track reconstruction}

The ATLAS inner detector [2] has forward-backward symmetry and is composed of three cylindrical sub-detectors which form concentric rings in the barrel, and parallel disks in the endcaps. The two innermost sub-detectors are the silicon pixel detector and the silicon microstrip detector (SCT), which each have four barrel layers and respectively have three and nine endcap disks on each side. The outermost sub-dectector is the transition radiation tracker (TRT), composed of straw tubes which run parallel to the beamline in the barrel, and extend radially outward in the endcap.

In the ATLAS inner detector, standard tracking takes place in two steps. The first step, referred to as inside-out tracking looks for track seeds of three space points in the silicon detectors. Track candidates are then formed based on the track seeds that meet certain criteria, using a combinatorial Kalman filter [3]. These track candidates are then fed through an ambiguity solver, and finally those that pass are attempted to be extended into the TRT. The second step, known as outside-in tracking, instead seeds tracks in the TRT and extends them backward towards the IP. Tracks formed in both the inside-out and outside-in tracking passes have fairly strict requirements placed on their longitudinal $\left(d_{0}\right)$ and transverse $\left(z_{0}\right)$ impact parameters, as well as on the maximum number of shared silicon modules; these requirements reduce both the CPU time and the fake rate of standard tracking, but limit the ability to reconstruct tracks from long lived particles.

A third tracking pass is used to reconstruct tracks with larger impact parameters than the stringent criteria of the standard tracking passes are equipped to handle. This third large radius tracking pass operates much in the same way as inside-out tracking, by forming track seeds from three space points in the silicon detectors and then extending track candidates outward, using the hits which are leftover from the standard tracking passes. In the large radius tracking pass, however, the seed extension uses a sequential rather than a combinatorial Kalman filter. The large radius tracking pass also imposes much less strict requirements on $d_{0}$ and $z_{0}$, as well as on the maximum $\eta$, and maximum number of shared silicon modules per track. While the much looser requirements on the tracks means that this large radius tracking pass has a much higher fake rate than the standard tracking pass, it also allows for the reconstruction of displaced tracks with high efficiency.

\section{Samples used for validation}

For the validation of the large radius tracking pass, two different monte carlo (MC) signal sam- 
ples were used. One was based on an R-parity violating SUSY model which ultimately produced displaced leptons in the inner detector, while the second was based on a Split SUSY R-hadron model which produced displaced hadrons in the inner detector. These models were chosen to be complementary in their output - both leptons and hadrons - as well as in their decay particles' production radii $\left(r_{\text {prod }}\right)$ and $p_{\mathrm{T}}$ spectra.

In addition to the signal MC samples, a minimum bias MC sample and a minimum bias data sample were used to compare the performance of the large radius tracking pass on simulation and data.

\section{Tracking efficiency}

The track reconstruction efficiency in the signal MC samples was evaluated using two different methods. The first was to use an efficiency in which the denominator consisted of all of the charged particles from the MC simulation which passed a simple set of fiducial selections and had parents which were the long lived particles (referred to here as signal truth), and the numerator was tracks that were matched to the signal truth particles with more than 50\% matching probability, split into categories of tracks from the standard tracking pass, tracks from the large radius tracking pass, and the combined standard and large radius track collection. As seen in figure 1(a), the efficiency of the combined track collection is nearly $100 \%$ at very small $r_{\text {prod }}$ for the charged particles, and falls steadily as $r_{\text {prod }}$ increases to $300 \mathrm{~mm}$, with the contribution from the large radius tracking pass making a significant improvement to the overall efficiency after $r_{\text {prod }}$ of about $20 \mathrm{~mm}$.

The second type of efficiency considered, referred to here as a technical efficiency, utilizes slightly

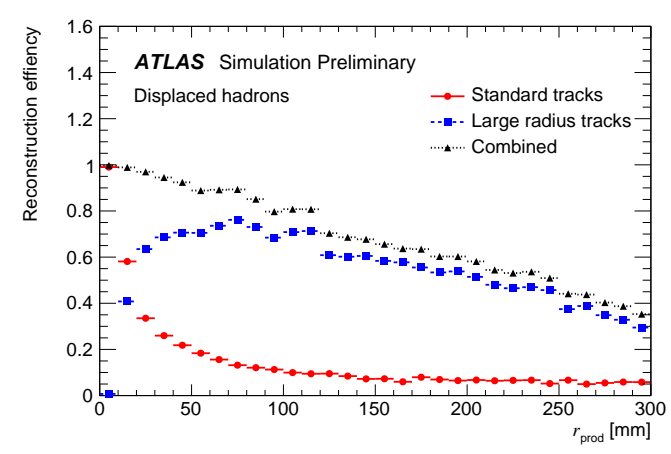

(a) Reconstruction efficiency vs $r_{\text {prod }}$

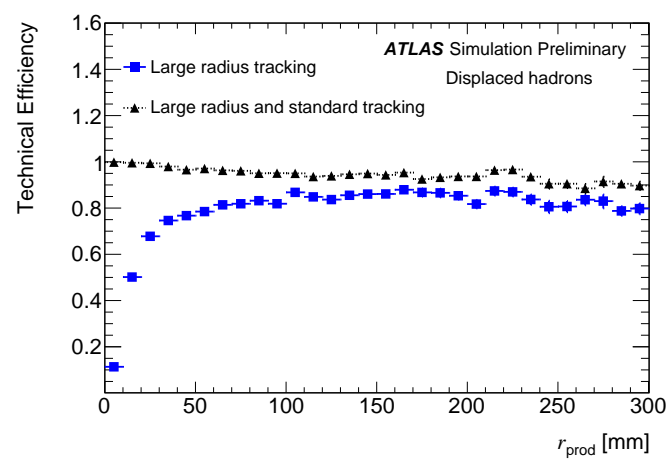

(b) Technical efficiency vs $r_{\text {prod }}$

Figure 1: The (a) efficiency and (b) technical efficiency for reconstructing signal truth particles vs $r_{\text {prod }}$, for the displaced hadrons sample (the displaced leptons sample, not shown, behaved similarly).

different criteria for the denominator, in this case only taking into account those signal truth particles which would have crossed enough layers in the silicon detectors such that their resulting tracks would have at least seven silicon hits (the minimum number needed for reconstruction). Using this method of evaluating the efficiency, figure 1(b) demonstrates that the total combined track collection has a technical efficiency of $90 \%$ or more for $r_{\text {prod }}$ out to $300 \mathrm{~mm}$, with most of that efficiency resulting from the large radius tracking pass beyond a $r_{\text {prod }}$ of about $20 \mathrm{~mm}$. This method of evaluation demonstrates that with the addition of the large radius tracking, most long lived particles that can be reconstructed, will be. 


\section{Track quality and robustness against pileup}

The nature of the large radius tracking pass leads to a high rate of poor quality tracks in addition to the very high technical efficiency. Several studies were undertaken to find improvements that could be made to the tracking to cut down the number of poor quality tracks while having a minimal impact on the efficiency. It was found that placing somewhat stricter requirements on the number of TRT hits and on the TRT precision hit fraction (a measurement of the quality of the TRT hits), as well as on the number of SCT hits (currently tracking considers the number of silicon hits, not the number of SCT hits specifically), would cut out regions of parameter space in which the majority of tracks are of poor quality, while cutting out few tracks which are matched to signal truth particles (see figure 2).

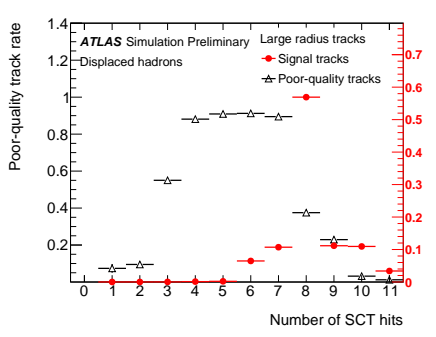

(a) Poor quality tracks and signal tracks vs number of SCT hits

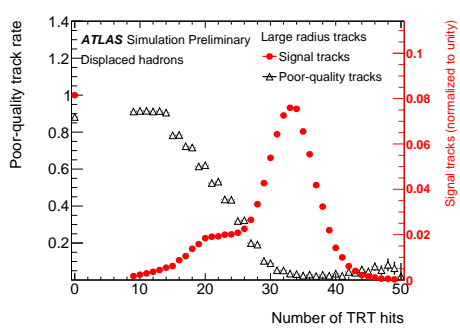

(b) Poor quality tracks and signal tracks vs number of TRT hits

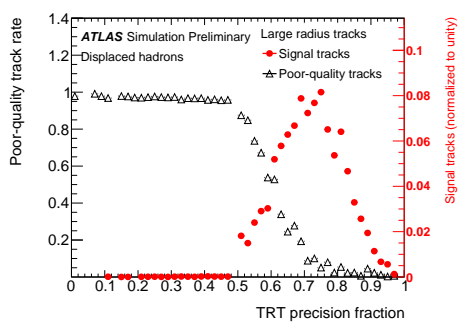

(c) Poor quality tracks and signal tracks vs TRT precision hit fraction

Figure 2: Rate of poor quality tracks vs the distribution of signal truth matched tracks as a function of (a) the number of SCT hits per track, (b) the number of TRT hits per track, and (c) the TRT precision hit fraction.

Additional studies concerned how the performance of the large radius tracking would be impacted by increased pileup $(\mu)$ - the number of proton-proton collisions in any given bunch crossing. The motivation behind examining the tracking performance vs $\mu$, is that $\mu$ is projected to increase throughout the rest of Run 2 and in Run 3. Two of these studies were presented at EPSHEP in 2017 - the trends of the standard deviation of the residual of $d_{0}$ and of the reconstruction efficiency (the non-technical efficiency) of the combined track collection vs $\mu$.

The first of these studies found that the resolution of $d_{0}$ is relatively flat as $\mu$ increases from 0 to 40 (figure 3(a)), with some slight degradation vs $\mu$ at large $r_{\text {prod. }}$. It was also found that the reconstruction efficiency for tracks with smaller $r_{\text {prod }}$ is fairly flat vs $\mu$ (figure 3(b)), but for large $r_{\text {prod }}$, the efficiency decreases as $\mu$ increases, indicating that further work on the large radius tracking pass is required to maintain high efficiency as $\mu$ increases in the future.

\section{Tracking performance on data vs MC samples}

In order to verify that the large radius tracking pass performs similarly on data and on MC samples, a study was done using minimum bias MC samples and data selected with a minimum bias trigger in low $\mu$ conditions in the ATLAS detector. The displaced particles in these samples are primarily due to hadronic interactions with the detector material. The tracks in the MC samples were weighted such the the $p_{\mathrm{T}}, \eta$, and kinematic distributions matched between the data and MC 


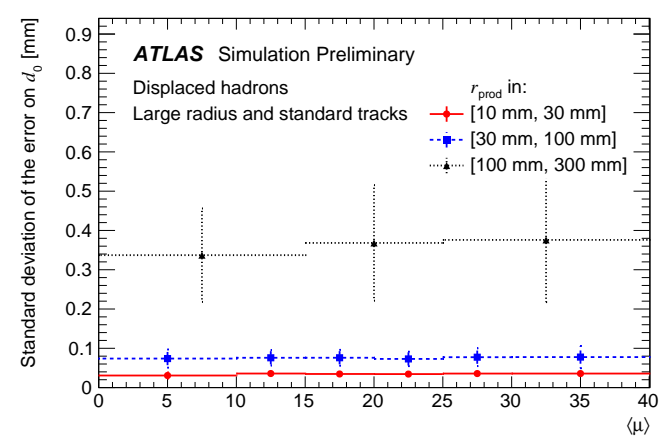

(a) Standard deviation of the residual of $d_{0}$ vs $\mu$

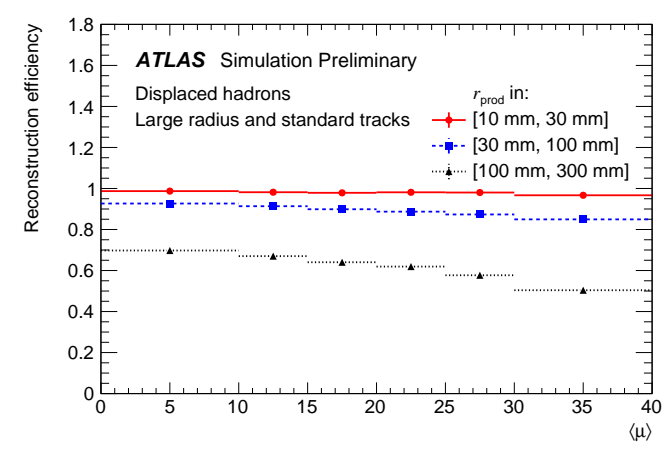

(b) Reconstruction efficiency vs $\mu$

Figure 3: Performance vs $\mu$ of (a) the standard deviation of the $d_{0}$ residual (referred to on the y-axis as the standard deviation of the error), and (b) non-technical reconstruction efficiency.

samples. The study demonstrated very good agreement between the distribution of the number of tracks in each sub-detector, the distributions of the average number of hits per sub-detector vs $\eta$, and the distributions of various tracking parameters, such as the radius of the first hit on the track (figure 4), with slight discrepancies primarily coming from some non-uniformities in the detector which weren't modeled properly in the simulation.

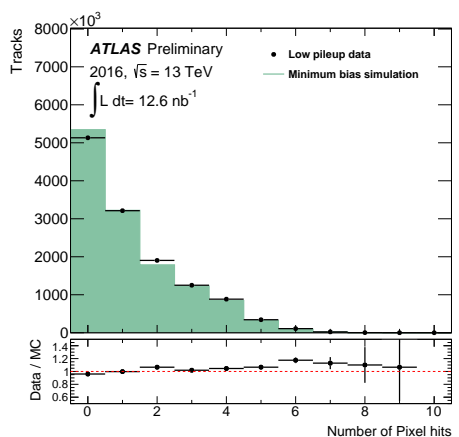

(a) Number of pixel hits distribution for data and $\mathrm{MC}$

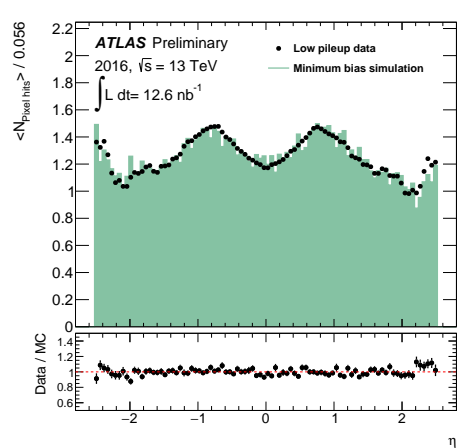

(b) Average number of pixel hits vs $\eta$ distribution for data and $\mathrm{MC}$

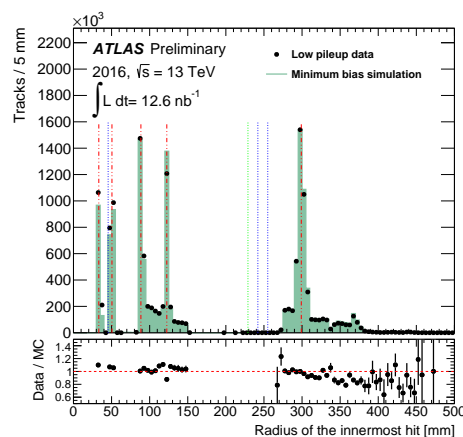

(c) Radius of the first hit on the track distribution for data and MC

Figure 4: Data vs simulation comparisons for (a) number of pixel hits, (b) average number of pixel hits vs $\eta$, and (c) radius of first hit on the track (the colored dashed lines represent detector related material).

\section{Conclusion}

A third tracking pass is implemented in ATLAS track reconstruction to assist in the reconstruction of tracks from displaced decays. While this tracking pass has an inherently high rate of poor quality tracks, the addition of tracks from this pass to the overall track collection allows for a very high reconstruction efficiency of those tracks which pass through a minimum number of silicon layers. This tracking pass performs consistently on data and MC samples. Future work will 
be undertaken to try to reduce the fake rate, and to ensure that the performance does not degrade as the pileup in collisions in the ATLAS detector increases.

\section{References}

[1] ATLAS Collaboration, Performance of the reconstruction of large impact parameter tracks in the inner detector of ATLAS, ATL-PHYS-PUB-2017-014, http://cds.cern.ch/record/2275635 (2017)

[2] ATLAS Collaboration, The ATLAS Experiment at the CERN Large Hadron Collider, JINST3 (2008) S08003, https://cds.cern.ch/record/1129811

[3] ATLAS Collaboration, Performance of the ATLAS Silicon Pattern Recognition Algorithm in Data and Simulation at $\sqrt{s}=7 \mathrm{TeV}$, ATLAS-CONF-2010-072, https://cds.cern.ch/record/1281363 (2010) 